\title{
Development of a 3D-Printed Bionic Hand with Muscle- and Force Control
}

\author{
Florian Dannereder ${ }^{1}$, Paul Herwig Pachschwöll ${ }^{1}$, Mohamed Aburaia ${ }^{2}$, Erich Markl ${ }^{2}$, \\ Maximilian Lackner ${ }^{2}$, Corinna Engelhardt-Nowitzki ${ }^{2}$ and Diane Shooman ${ }^{2}$
}

\begin{abstract}
The majority of people with upper extremity loss replace their arm and hand with a low-cost prosthesis. However, an average prosthesis only covers minimal functionality in comparison to a human hand, and the user is strongly limited in everyday life. Sophisticated bionic hands have been developed to replace upper extremity functionality. A bionic hand can be controlled via muscle contraction of the upper extremity or the shoulder area, and can replace the main functions that a human needs in everyday life. Nearly every hand movement and the independent movement of the fingers can be produced through a rotation mechanism around the wearer's wrist. Since these bionic hands are very expensive, only a small percentage of the world population have the privilege to own one. To close the gap between customer, designer and engineer, an open source bionic hand that can be 3D-printed is a cost effective possibility. The result of this project is a cost effective 3D-printed bionic hand that can be reprogrammed for user specific functions. The sensed muscle regions can be changed spontaneously as needed. The sensitivity of the muscle contraction and the gripping force are adjusted by software using a closed loop control.
\end{abstract}

\section{INTRODUCTION}

Mastering the use of a bionic hand to manipulate objects in our daily environment can be so complex, that numerous of users revert back to simpler prosthetics. A particular technical challenge in bionic hand design is to create an effective interface for the wearer, and to provide a wide spectrum of grip types through muscle control. Individual differences in each human body influence the control algorithms and the muscle contraction detection. To improve the daily use, some personal settings e.g. different speeds, thresholds or grips should be adjustable. This paper describes a 3D-printed bionic hand with 15 different gripping styles, which can be controlled by muscle contraction from the upper extremity. It provides an automatic stop of the finger movement when touching an object at a determined force, although the users muscle is still contracted. This simplifies the bionic hand control through muscle contraction and has a tremendous impact on controllability.

\section{STATE OF THE ART}

Modern bionic hands are controlled by myoelectric signals, which allow precise control of different grips. Those

\footnotetext{
${ }^{1}$ Florian Dannereder and Paul Herwig Pachschwöll are students of the study program Mechatronics/Robotics at the University of Applied Sciences Technikum Wien, Austria fflorian.dannereder, paul_herwig.pachschwoell\}@technikum-wien.at

${ }^{2}$ Mohamed Aburaia, Erich Markl, Maximilian Lackner, Corinna Engelhardt-Nowitzki and Diane Shooman are with the department of Advanced Engineering Technologies at the University of Applied Sciences Technikum Wien, Austria \{aburaia, markl, lacknerm, engelhac, shooman\}atechnikum-wien.at
}

myoelectric signals sense a chosen muscle region that is contracted by the prosthesis user. With this method the amputees brain is capable of controlling the bionic hand with good accuracy and low difficulty [1]. Currently, the most popular commercial bionic prostheses with high-technical functionality are the Touch Bionics I-Limb-Ultra and the Bebionics RSL Steeper. This two bionic hands, shown in Figure 1, will be discussed in this chapter.
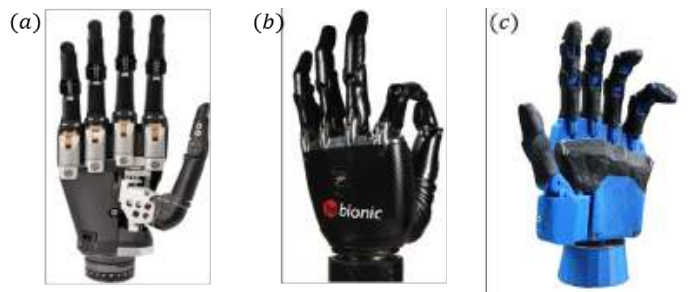

Fig. 1. High technical bionic hands (a) Touchbionics iLimb Ultra [2], (b) RSL Steeper Bebionic [3]

High technical bionic hands, which can replace upper extremity functionality, are realized with eleven joints. In comparison, a real human hand has 33 joints [4]. The most bionic hands facilitate a finger movement with a coupler mechanism, or with a tendon linkage. The eleventh joint is the thumb slewing mechanism, to change between an open and a closed hand. Different finger mechanisms with up to two joints are shown in Figure 2.

An important characteristic of the finger construction is a self-locking mechanism, which can be carried out in different ways. A self-locking mechanism is important for the end positions of the fingers, to prevent some inadvertent position change of the current activated grip. The I-Limb Ultra uses a DC motor with a spur gear, to transfer the torque to a worm gear. In contrast, the RSL Steeper uses a linear DC motor with an integrated lead screw. This makes it possible for both bionic hands to block the finger-movement while the motors are turned off. In Table I different technical specifications are shown.

\section{PROBLEMS AND CHANCES}

The typical muscles of a prosthesis wearers forearm are not always useable, which means that the position of the electrodes has to be selectable. In case of only one useable forearm muscle, a shoulder or an upper arm muscle can 
TABLE I

TECHNiCAL SPECIFICATIONS OF THE I-Limb ULTRA AND THE RSL STEEPER [5]

\begin{tabular}{|l|l|l|}
\hline Product & I Limb Ultra & RSL Steeper \\
\hline Developer & Touch Bionics & Otto Bock \\
\hline Weight & $405-479 \mathrm{~g}$ & $495-539 \mathrm{~g}$ \\
\hline Number of Joints & 11 & 11 \\
\hline Number of Actuators & $5+1$ (motorized thumb) & 5 \\
\hline Actuation Method & DC Motor-Worm Gear & Linear DC Motor-Lead Screw \\
\hline Joint Coupling Method & Tendon Coupling & Coupler mechanism \\
\hline
\end{tabular}

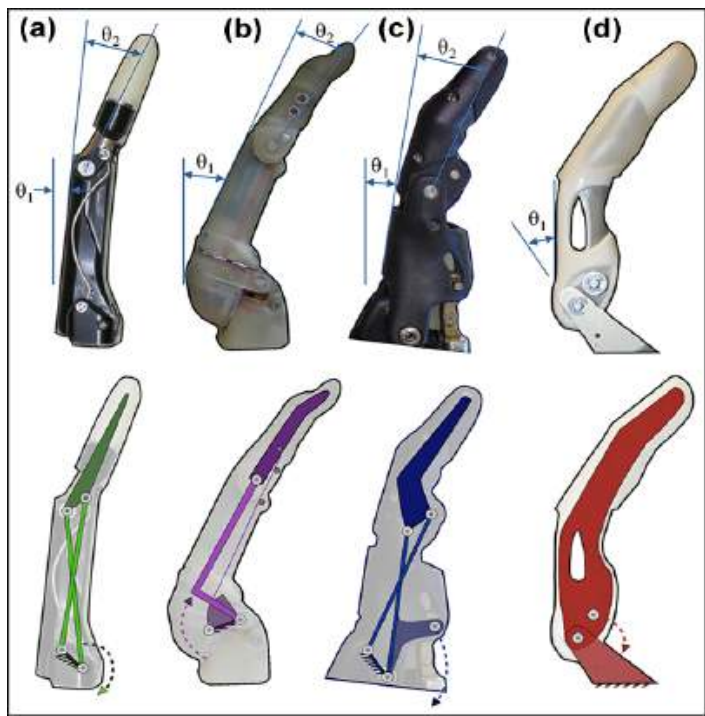

Fig. 2. Different finger mechanisms used for bionic hands with up to two degrees of freedom, (a) Vincent, (b) I-Limb, (c) RSL Steeper, (d) Michelangelo [5]

be used instead. The orientation of the bionic hand before picking up an object is also important. The only way to do so is through a rotation made by the bionic hands wrist, which adds another degree of freedom. This function is used to tilt a bottle and fill a cup. To achieve a tight grip on the bottle, every finger is powered by an actuator, which allows an independent finger movement. To switch between an open hand and a grip for taking a bottle, a slewing thumb is necessary. The most common grips do not always need every finger, therefore some precision grips have been programmed. Picking up a pencil from a table can be done with the use of three fingers. To avoid a vibrating and noisy bionic hand, self-locking actuators have been combined with a coupler mechanism. The bionic hands actuators create a high force, which create the necessity of a force control when the hand closes, to avoid damaging itself or the objects gripped by the fingers.

\section{A. Placement of the Muscle Sensors}

Interpretation of sensed muscle contraction is a complex procedure. Somehow, when a muscle is contracted, the opposite muscle contracts softly too. This creates the possibility of using different electrode modes, like a single mode or a dual mode. Raw detected muscle signals with a total of 105 measurement points on the $\mathrm{x}$-axis, recorded in a time of two seconds is shown in Figure 3. The y-axis represents the 10-bit ADC-value from the Myoware muscle sensors. For the data point evaluation, a threshold has to be set, which defines whether the muscle was seriously contracted or not. If a digital value of more than 160 were interpreted as a positive muscle contraction, every signal jump exceeding a threshold of 160 would be read as a single detected muscle impulse. This makes toggling functions by using muscle contraction difficult. The measurement points 92, 97 and 102 show fast signal jumps, with a severe influence on the muscle contraction detection [6]. These three short impulses are the result of contracting the opposite muscle region.

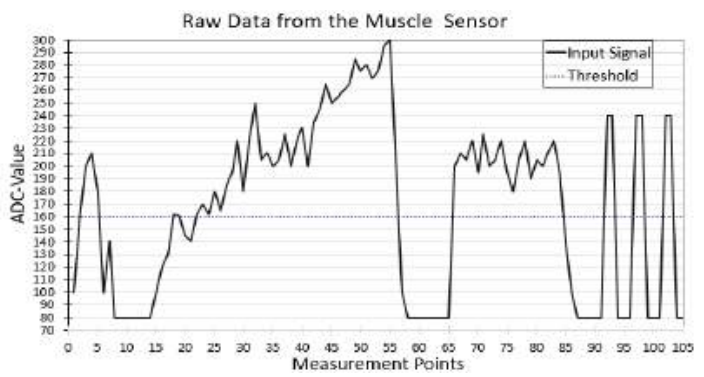

Fig. 3. Recorded signal of two short muscle contractions of the forearm, converted into digital values

To avoid a false detection, a filter is the best solution, nevertheless it is possible to attach the electrode to another muscle region. Another common muscle region is the shoulder area, which cannot be influenced by the forearm muscles. Concerning the fact that almost every muscle can be detected, it is possible to connect the bionic hand with another muscle electrode. A threshold of an ADC-value of more than 160 would create the following interpretation of the muscle activity. 


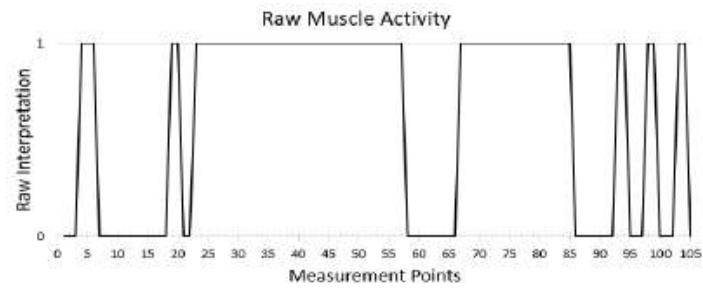

Fig. 4. Using a threshold of 160 to decide if a muscle is contracted or not $(0=$ relaxed, $1=$ contracted $)$

\section{B. Independent Finger and Wrist Movement}

Without a rotating wrist, the shoulder joint would be the only way to adjust the orientation of the bionic hand. Small jobs like filling a liquid into a cup would become very difficult without an additional degree of freedom. Grabbing a bottle needs an encircling thumb that ensures a steady grip [7]. A bionic hand with an independent finger movement can be used for many different grips, which makes it very useful in everyday scenarios. The coupler mechanism makes it possible to transform the actuators linear movement into a finger movement that keeps the relation between its travelled distance and the position of the fingers. The necessary force to close the hand is not constant and changes while closing. A measurement of the idle closing current of each finger involves further details. The actuator rod travels $13.5 \mathrm{~mm}$ to convert the open palm into a closed palm with every finger in its bend position, which is explained in Figure 10. This delivers an amount of measurable steps for the force control, which monitors the force by finger movement. The current is not steady, which makes the use of a simple current limit inaccurate. A method had to be developed to use the collected current data and create a more accurate force control. Nevertheless, each finger has its own current progress, so the created method has to be flexible.

\section{METHODS}

The 3D-printed bionic hand is an open-source device made only of nonindustrial components to ensure that every interested person is able to reconstruct it. The microcontroller is an Arduino Uno, which realises 15 different gripping styles. The movement of the fingers is made with linear actuators, which create an independent finger movement. To keep a human-like shape the bionic hand has five fingers, a rotating wrist and a pivoting thumb. The prosthesis is controlled by muscle activity and allows a high usability.

\section{A. Implementation of a Filter for Muscle Noise Reduction}

The bionic hand is designed to be controlled by two muscle electrodes. The usability as a forearm prosthesis makes using the forearm muscles the obvious choice. The placement of the electrode has an important role for the controllability. Contraction of the opposite muscle occurs spontaneously, which makes it hard to differentiate between a seriously and a spontaneously contracted muscle. The muscle signal displayed in Figure 5 shows two short muscle contractions with small signal jumps. The easiest way for smoothing those irregularities would be a first order low pass filter [8]. The drawback of using circuits is that the signal is stored in a component, which changes the speed of the signal processing significantly. Each finger would need to be equipped with such a circuit, nevertheless space inside a bionic hand is limited. Therefore, a digital solution was created. Another benefit of a digital solution is that a special behaviour can be forced. Processing the raw muscle signal from the electrodes can then be optimised to react differently on a rising signal rather than on a falling edge. This enables integration of the raw signal to create a noise reduction but which edges off the signal if a level drop is measured. The following figure shows the raw signal from Figure 3 explained in Chapter III-A, which has been filtered for better controllability of the bionic hand.

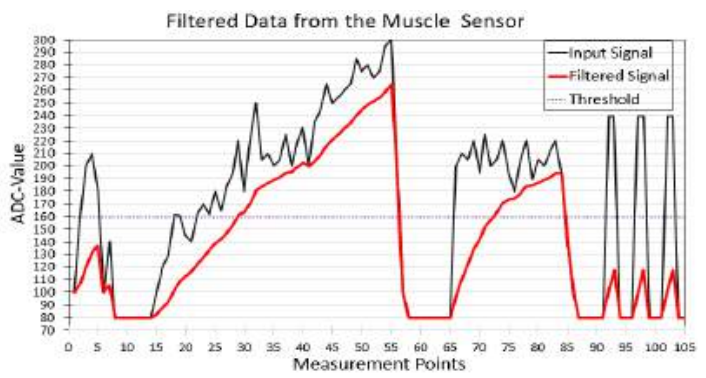

Fig. 5. Using a threshold of 160 to decide if a muscle is contracted or not $(0=$ relaxed, $1=$ contracted $)$

A closer look at the figure shown above makes it clear that the similarity of the signals is still given. The digital filter was optimized to delete narrow and high jumps without deformation of the signal sequence. The mentioned edge detection allowed a fast adjustment on falling edges. The following figure shows the interpretation of the filtered signal.

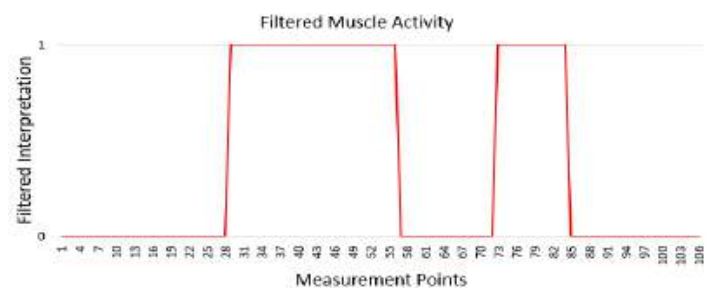

Fig. 6. Interpretation of the digitally filtered signal of two short muscle contractions by using a threshold of $160(0=$ relaxed, $1=$ contracted $)$

Compared to Figure 4 (Chapter III-A) the difference is easily noticeable, and a better controllability of the bionic hand was achieved. 


\section{B. Estimation of the Gripping Force}

Calculation of the gripping force has to be fast, applicable for every finger, and with low computing consumption. The force transfer of the actuator to the finger is a linear translation, which makes creating an accurate mathematical formula possible. A lookup table of every fingers idle current was recorded and used instead. With this individual information, the position related current limit was defined as the idle current increased by 35 percent. This method turned out to be precise enough, regardless of the position of the actuator or finger. Related to the different finger size, the relation between actuator current and finger force is individual for every finger, so the thresholds were finger-size related too. With this method the current limit shown below was defined and used for finger.

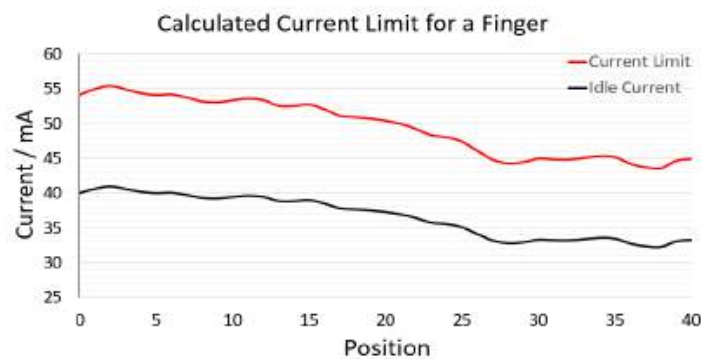

Fig. 7. Calculated current limit for a finger by using a current increase of 35 percent to create a specific current limit

Tests have confirmed that a 35 percent increase is enough to ensure a tight and reliable grip. This factor is adjustable and can be set specific for a grip.

\section{Construction}

As seen in Figure 8, the bionic hand has seven servomotors, which enables seven degrees of freedom. In each finger, an individual six-joint linkage is integrated, to perform a particular movement profile. The forearm is equipped with an extra motor for the wrist rotation, such as the controller and additional electronic components. In the next two sections, the realization of the finger movement and the thumb slewing mechanism will be explained in detail.

1) Finger Mechanims: For the construction, a few necessary requirements must be considered. To ensure that the bionic hand supports a two-joint finger movement, a sixjoint linkage is integrated in each finger. The six-joint linkage provide a finger movement with two constrained angles $\theta_{1}$ and $\theta_{2}$. Therefore, a real human finger movement can be reproduced. Figure 9 shows a retracted and an extended finger position with the constrained angles $\theta_{1}$ and $\theta_{2}$.

The reason for using a linkage was, that it is possible to combine it with a self-locking linear servo motor. The motor is the PQ12-R micro linear servo motor from Actuonix, with a total stoke of $20 \mathrm{~mm}$, and a maximal linear force of $50 \mathrm{~N}$ [9]. The linear servo motor will fully retract the motor

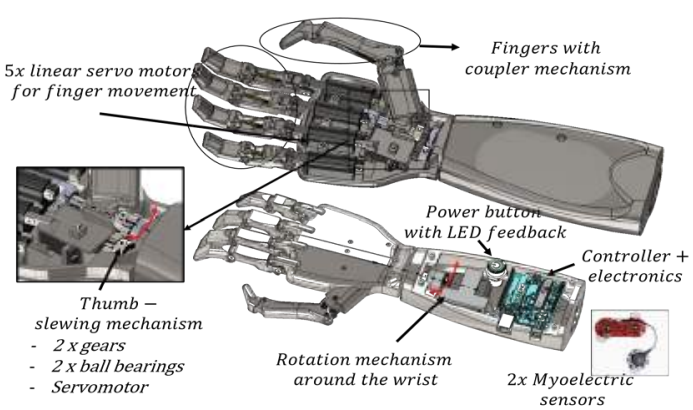

Fig. 8. CAD-Model

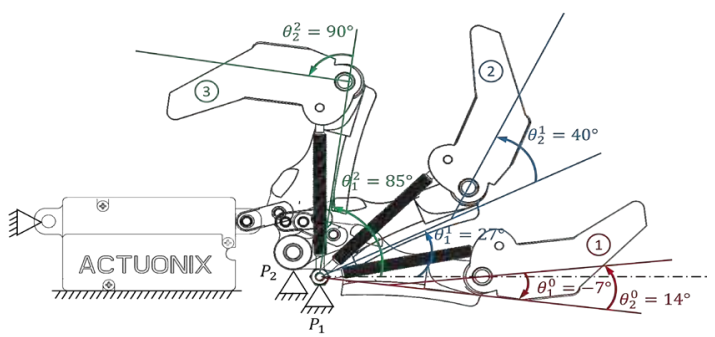

Fig. 9. Two joint finger movement with the constrained angles and, realized with a six-joint finger linkage

shaft with a $2.0 \mathrm{~ms}$ pulse signal, and a $1.0 \mathrm{~ms}$ pulse signal will fully extend the motor shaft. Therefore, every position from $0 \mathrm{~mm}$ to $20 \mathrm{~mm}$ is approachable with the associated pulse signal [10]. The self-locking mechanism is a necessary requirement for the end positions of the fingers. The fingerlinkage is constrained via a two-joint couple to the motor shaft, and therefore different finger positions by the linear movement of the motor shaft is accessible. The finger joints rotate around the instantaneous center (IC) of rotation $\left(\mathrm{P}_{1}\right.$, $\mathrm{P}_{2}$ ), which are mounted into a fixed bearing inside the hand cover. The motor is also fixed inside the hand cover, and the motor shaft can move linearly.

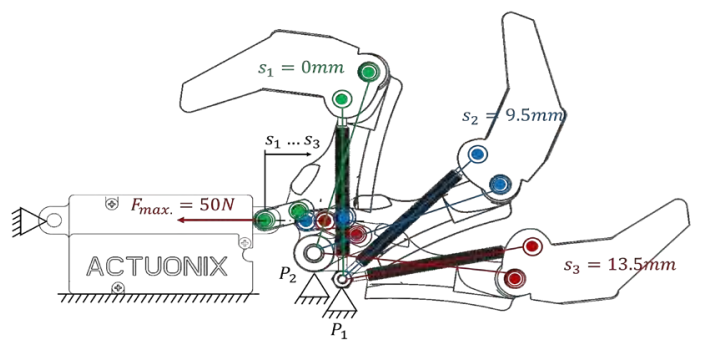

Fig. 10. Used coupler mechanism for finger movement with a total motor stroke of $13.5 \mathrm{~mm}$ 
The integrated motor type is called MG90S, and is a micro servo motor with a torque of $0.1962 \mathrm{Nm}$ [11]. It is connected with a spur gear, which transfers the torque to the rotation axis of the thumb. The rotation axis of the thumb meshes with another gear, and is mounted with two ball bearings (see Figure 11).

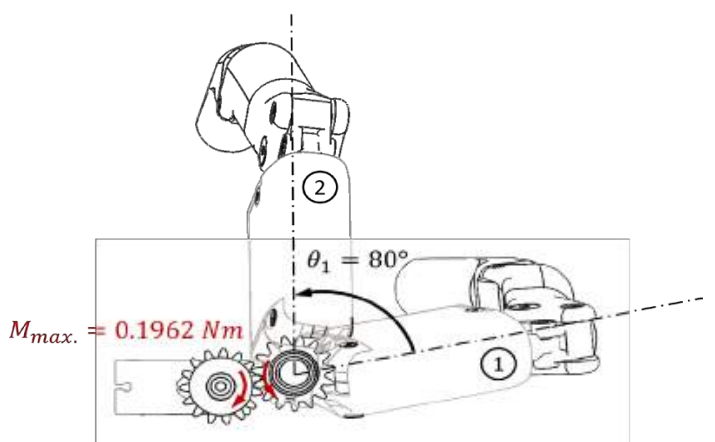

Fig. 11. Used coupler mechanism for finger movement with a total motor stroke of $13.5 \mathrm{~mm}$

\section{RESULTS}

To find possible grips for programming the bionic hand, the force feedback was the first implemented function. This enabled the testing of different daily objects inside the bionic hands palm. Based on test scenarios a total set of 15 different grips has been programmed. The first tests were conducted without the use of muscle sensors, mechanical switches were used instead. Later on, the muscle sensors and its noise cancelling functions were added, which made the bionic hand controllable by muscle contraction. The way each grip is used is different, therefore the speeds for operating the fingers have been adjusted as well. The controllability of the bionic hand is precise enough to pick up a small resistor from the table surface. The used controller has still some pins left for further extensions and approximately 15 percent of its memory is available. The placement of the muscle sensors can be chosen arbitrarily, depending on the chosen muscle areas. The independent finger movement allows grips that cover up to 85 percent of the commonly used gripping scenarios.

\section{A. A fully assembled 3D-printed bionic Hand}

The final assembled bionic hand can be seen in Figure 12.

The 3D-printed bionic hand can be separated into three main assembly parts. The first assembly parts are the 3Dprinted components. 3D-printed components are for example the fingers, different covers and other components designed special for this bionic hand, and are printed with a selective laser sintering-printer. In sum, the bionic hand consists of 27 different 3D-printed parts. The second set of assembly parts were purchased, these are components like ball bearings, gears, motors, the muscle sensors and the controller. In sum,

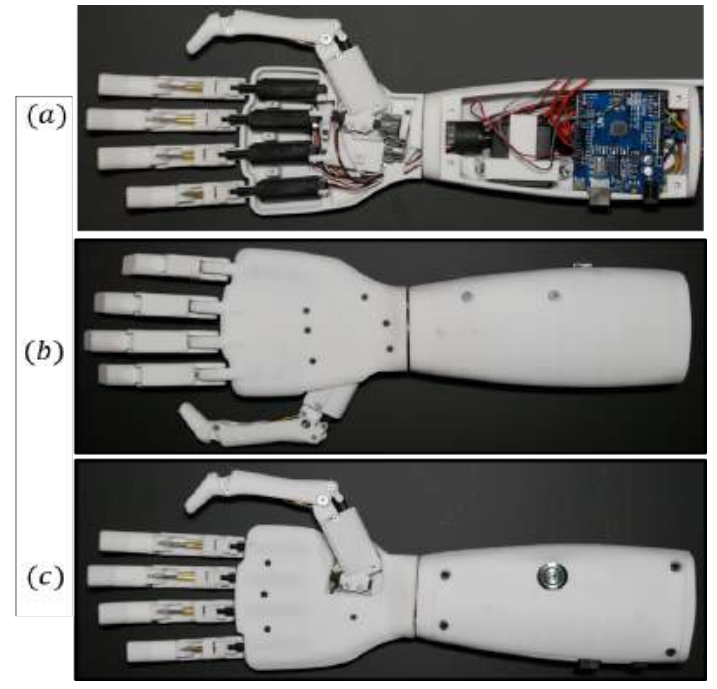

Fig. 12. Fully assembled 3D-printed bionic hand, (a) Assembled bionic hand without cover, (b) Topview internal side, (c) Topview external side

22 different components were purchased for this project. The last assembly parts are 159 screw-elements, like nuts, shells and washers.

\section{B. Gripping Styles}

Typical daily grips have been programmed and adjusted to be controlled with muscle sensors. The bionic hands 15 predefined grips use force feedback to ensure a tight grip on the taken object. Figure 13 displays a map of the predefined grips. The hand positions indicate the available functions in the function 1, function 2 or function 3 layers. The advantage of independent fingers makes it possible to create all kind of grips. Therefore, some functions close the index-finger for locating the object in the hand before the other fingers close. Another special grip is the anti-slip grip, using the small finger to prevent slippery objects from sliding out of the hand. Other objects require the parallel grips which close all finger in a parallel formation. To pick up thin objects like a tissue pack, the precision grips have been implemented. They move only the thumb and index-finger, the wearer decides which will be the moving finger. The keyboard grip enables the index-finger to point at something or to press a button. It is also possible to close this finger to activate the key grip, which is perfect for taking a ticket from a parking ticket machine. Small objects do not need contact to every finger, which makes moving them unnecessary. Therefore the tripod grips moves only the thumb, index- and middle finger. The "pen" grip is a particularly advancement, enabling the wear to use a pen for drawing or writing. The bionic hand is a device that can be used to get back to your hobbies. Therefore, the extreme grips have been designed. They use 
the edge of the finger tips to pick up resistors, cables or nails. The hook grip is a more robust grip for lifting heavy objects of up to three and a half kilograms, because the fingers are aligned to counterbalance the weight.

\section{Scenarios}

The 15 programmed gripping styles were tested in different scenarios, which show the application range of the bionic hand. Figure 15 shows different simple gripping examples, without an interaction of the left human hand, carried out by muscle contraction of the right forearm. Other examples with a left human hand interaction are shown in Figure 14. An important specification for grabbing an object is the closed loop control of the linear actuators, explained in Chapter IVB.

\section{1) Simple Grips without Human Interaction:}

a) Normal: The first example shows the normal grip, used to grab the cap of a can. The big advantage in this example is the closed loop control, by which the finger movement of the bionic hand will automatically stop after grabbing the object.

b) Precision: Example (b) shows a precision grip, where the inside of the forefinger touches the front side of the thumb.

c) Normal: The third example shows the normal grip again. Grabbing a small ball is a good example for demonstrating the finger positions. Each finger will move as long as the actuator load is lower than the determined value. Therefore, it is possible to grab objects of different shapes such as a ball.

d) Precision: This scenario shows the same precision grip again, this time with a fragile object.

e) Pen: To fix a pen between the forefinger, middle finger and the thumb, the pen grip can be used. Because of the integrated 6-joint linkage, combined with the self-locking actuators, the mechanical construction of the fingers is stable enough to perform a safe mount of a pen.

f) Hook: The hook grip can be used to lift heavy objects like toolboxes or shopping bags, with a maximum weight of three and a half kilograms. The self-locking actuators will prevent an inadvertent finger movement, while grabbing. Example (f) shows a toolbox whit a total weight of $3.5 \mathrm{~kg}$.

\section{2) Simple Grips with Human Hand Interaction:}

a) Precision: This example shows a match, fixed between the forefinger and the thumb of the bionic hand. The difficult part in this example starts when the wearer attempts to light the match with the matchbox. At this point, some additional forces act on the match, and therefore it is possible that the match slips away. This example demonstrates that the mechanical requirements are given, to fix an object with two fingers safely without it slipping when an external force interacts.

b) Precision: In this example another precision grip, where the fingertips are touching is used. This grip can be used to fix small objects like a resistor or a paper. c) Anti-Slip Normal: It is possible to enter the rotation mode from each grip. An integrated wrist rotation will replace the forearm rotation of a human hand. The anti-slip normal grip is a special grip for objects like a bottle. The little finger is in a retracted position and therefore it prevents objects from slipping through. After closing the fingers, the bottle is fixed enough to open the cap with the left human hand. To fill the liquid into a glass, the rotation mode can be activated to rotate the wrist for approximately 90 degrees. (a)

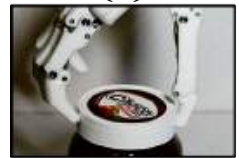

(d)

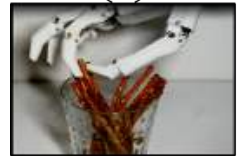

(b)

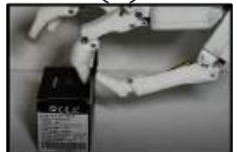

(e)

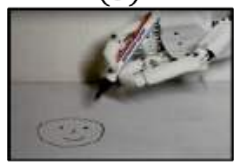

(c)

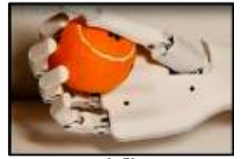

$(f)$

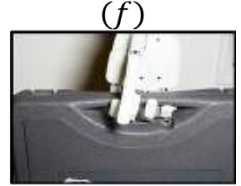

Fig. 13. Overview of 15 programmed gripping styles

(a)

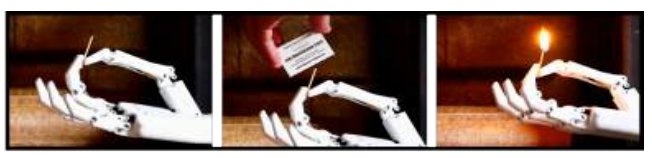

(b)

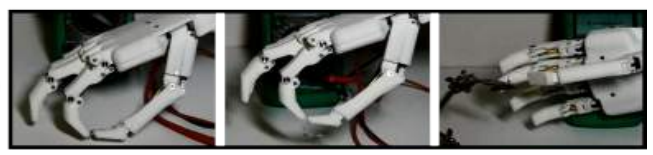

(c)

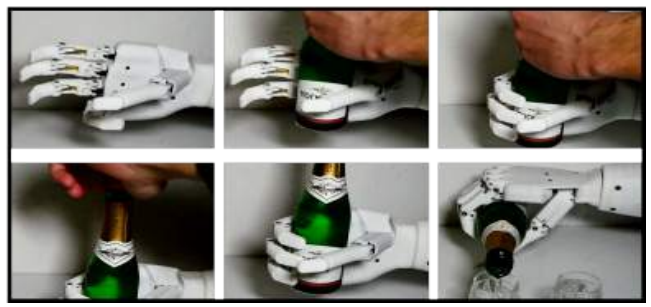

Fig. 14. Difficult gripping examples with an interaction of a left human hand

\section{CONCLUSIONS}

The methods demonstrated have been used to develop a bionic hand using linear actuators to move five independent fingers. For increased usability, a rotating wrist and a motorized slewing thumb were implemented. Typical daily grips have been programmed, tested and adjusted to be controlled with on-skin muscle sensors. These sensors have been placed 


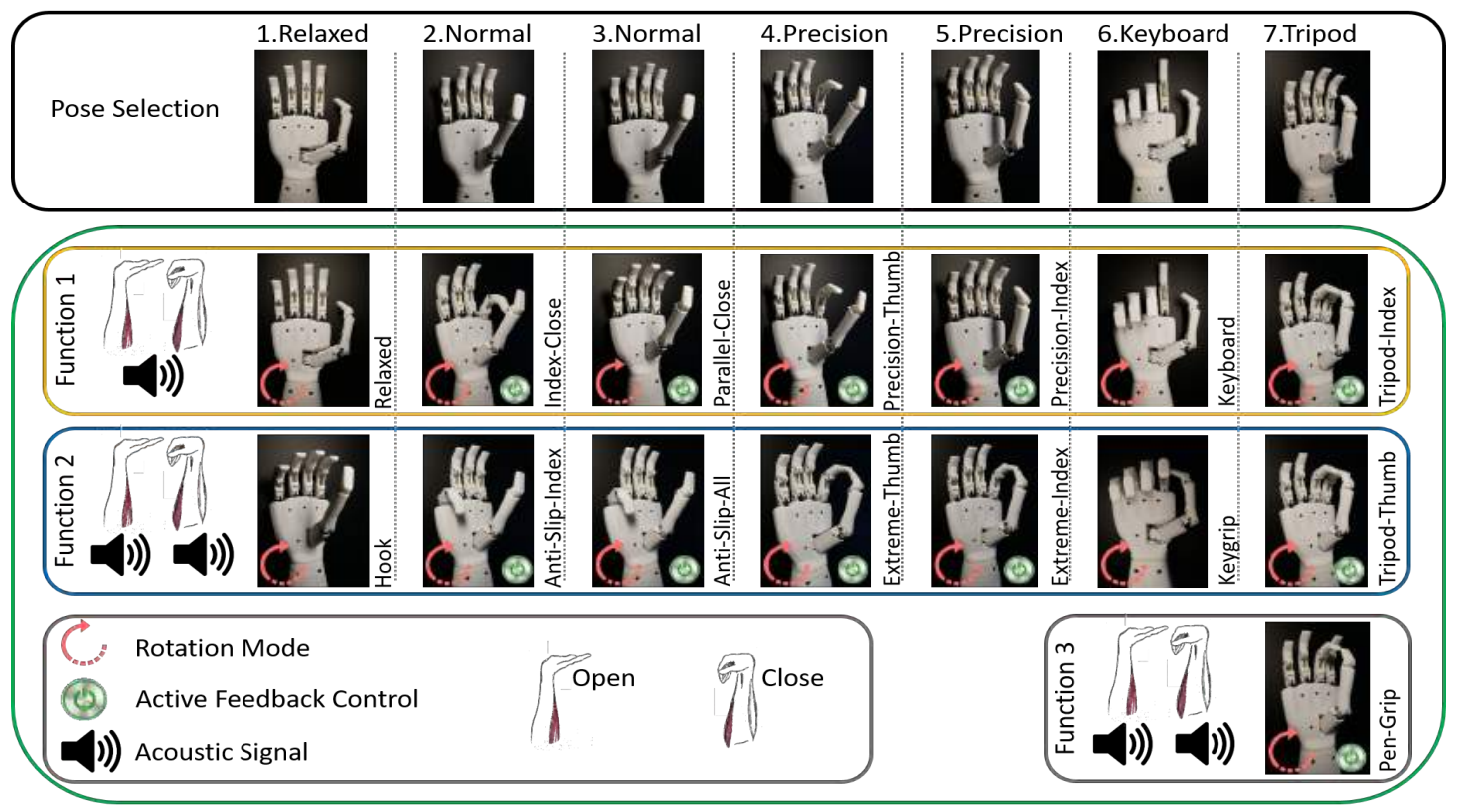

Fig. 15. Different daily lives gripping examples

on the forearm muscles, enabling the wearer to switch between different grips, rotate the wrist and control the fingers precisely. Tests of these muscle sensors revealed that some additional noise cancelling was necessary for interpretation of the muscle contraction. Therefore, a digital filter with a low pass characteristic was only used to smooth the rising muscle signal, a falling edge remained unmodified. This enhanced the controllability of the bionic hand extremely, and fine finger movements are now possible. To ensure a thin palm, the linear actuators have been combined with a six joint finger-linkage. This created a defined relation between the actuator position and the position of the fingertip. Those couplings are realised with metal tie rods with little clearance to achieve a good repeatability. The thumbs linear actuator is mounted on a slewing finger base, which can be positioned by a servo motor. It is then possible to grab a bottle and hold it securely enough for it to be manipulated. A big advantage of this bionic hand is the use of a force feedback, which turned out to be very precise. A human hand rotates the hand by using the forearm, however the bionic hand copied this function with the integration of a servo motor which acts as a wrist and permits a rotation of 135 . The combination of the actuators and servo motors made it possible to define 15 different grips, which use force feedback and are precise enough to hold a small resistor or a thin piece of paper between the finger tips. The functionality of a rotating wrist is implemented in every function and allows a fast object manipulation. The controller, mounted into the forearm of the bionic hand was programmed, and about 85 percent of a $32 \mathrm{~kb}$ memory have been used. The load on the actuators has never exceeded 20 percent to avoid damage to a grasped object. Nevertheless, if the force control notifies resistance, a short muscle impulse enables the fingers to close incrementally.

\section{REFERENCES}

[1] G. C. Matrone, C. Cipriani, M. C. Carrozza, and G. Magenes, "Realtime myoelectric control of a multi-fingered hand prosthesis using principal components analysis," Journal of NeuroEngineering and Rehabilitation, vol. 9, no. 1, p. 40, Jun 2012.

[2] (2018) Touch Bionics: I-Limb Ultra. [Online]. Available: http://www.touchbionics.com/products/active-prostheses/i-limb-ultra

[3] (2018) Ottobock Grip Patterns. [Online]. Available: http://bebionic.com/the_hand/grip_patterns

[4] (2014) BBC: The incredible human hand and foot. [Online]. Available: http://www.bbc.com/news/science-environment-26224631

[5] J. T. Belter, J. L. Segil, A. M. Dollar, and R. F. Weir, "Mechanical design and performance specifications of anthropomorphic prosthetic hands: a review," J Rehabil Res Dev, vol. 50, no. 5, pp. 599-618, 2013.

[6] I.-D. Nicolae and P.-M. Nicolae, "Denoising highly distorted small currents in an environment with variable levels of noise," in Electromagnetic Compatibility \& Signal/Power Integrity (EMCSI), 2017 IEEE International Symposium on. IEEE, 2017, pp. 299-304.

[7] K. Or, M. Tomura, A. Schmitz, S. Funabashi, and S. Sugano, "Interpolation control posture design for in-hand manipulation," in System Integration (SII), 2015 IEEE/SICE International Symposium on. IEEE, 2015, pp. 187-192.

[8] D. G. Stănescu, M. E. Ardeleanu, and A. C. Stan, "Designing, simulation and testing of low current passive filters used in the didactic activity," in Modern Power Systems (MPS), 2017 International Conference on. IEEE, 2017, pp. 1-4. 
[9] (2016) Actuonix: Miniature Linear Motion Series (Datasheet). [Online]. Available: https://s3.amazonaws.com/actuonix/Actuonix+PQ12+Datasheet.pdf

[10] K. Sakata and H. Fujimoto, "Perfect tracking control of servo motor based on precise model with pwm hold and current loop," in Power
Conversion Conference-Nagoya, 2007. PCC'07. IEEE, 2007, pp. 1612-1617.

[11] (2017) Metal Gear Servo MG90S: Metal gear with one bearing (Datasheet). [Online]. Available: https://s3.amazonaws.com/actuonix/Actuonix+PQ12+Datasheet.pdf 